\title{
A Survey of Haptics in Serious Gaming
}

\author{
Shujie Deng ${ }^{1}$, Jian Chang ${ }^{1 *}$, Jian J. Zhang ${ }^{1}$ \\ ${ }^{1}$ National Centre for Computer Animation, The Media School, Bournemouth University, Fern \\ Barrow, Poole, Dorset BH12 5BB, UK \\ \{sdeng, jchang, jzhang\}@bournemouth.ac.uk
}

\begin{abstract}
Serious gaming often requires high level of realism for training and learning purposes. Haptic technology has been proved to be useful in many applications with an additional perception modality complementary to the audio and the vision. It provides novel user experience to enhance the immersion of virtual reality with a physical control-layer. This survey focuses on the haptic technology and its applications in serious gaming. Several categories of related applications are listed and discussed in details, primarily on haptics acts as cognitive aux and main component in serious games design. We categorize haptic devices into tactile, force feedback and hybrid ones to suit different haptic interfaces, followed by description of common haptic gadgets in gaming. Haptic modeling methods, in particular, available SDKs or libraries either for commercial or academic usage, are summarized. We also analyze the existing research difficulties and technology bottleneck with haptics and foresee the future research directions.
\end{abstract}

Keywords: Serious Games; Haptic Devices; Haptic Modeling; Force Feedback; Virtual Reality

\section{Introduction}

Serious gaming based on videogames has achieved great success in the past 20 years. The effectiveness of learning from videogames is that it will intrigue the motivation to spend time on the tasks so to master skills, the design elements as narrative context, rules, goals, rewards, multisensory cues and interactivity can also stimulate the desire of learning [1]. Virtual simulation also helps reduce the potential hazard of dangerous tasks, solves ethical issues and saves the cost. Various training applications featuring haptics have been developed as in medical [2] and rehabilitation [3] areas.

Haptic feedback has recently become an indispensable component in serious games. It provides an additional perception modality of touch, together with vision and audio to generate a more immersive user experience. Immersive learning environment and interaction require system stability and real-time feedback for all sensory 
including vision, audio and touch. Integration of haptics into serious gaming has brought great challenges for both hardware and software development. This paper provides a survey of haptic application in serious gaming, and current research status of haptic hardware and modeling that are available for videogames design.

Touch perception can be divided into cutaneous and kinesthetic sensations, so haptics are correspondingly composed of two parts of perceptual feedback, tactile feedback and force/torque feedback. Devices can be categorized by different feedbacks they provide. Based on the game concept and budgets estimation, a suitable device genre is selected. The specifications of devices that are normally taken into consideration include degree of freedom (DOF), degree of force feedback (DOFF), size of workspace, max force/torque, stiffness, compatibility and extensibility of software development kit (SDK) etc. Commercial oriented games usually have a priority of being economic/affordable. The low-cost solution is to embed vibrotactile actuator into game devices, such as gamepads, mobile phones, etc. However, it restricts the rendering of abundant and detailed haptic feedback into unitary vibration. Medical and pedagogic oriented applications take advantage of more expensive devices, and produce more delicate feedback.

Generally, with the learning and training purposes, the force/torque and tactile perception gained from haptics provide user with better cognition of how they have performed in the task and helps them improve their performance in a more intuitive and efficient way. Especially for tasks that rely largely on haptic feedback, merely visual feedback helps little with improving performance or even causes errors. For instance, endoscopic surgical training is extremely difficult to achieve expected results without haptic feedback [4]; applications that focus on improving motor skills are also the same. More concretely, users who are visually impaired or blind benefit mostly from auditory and haptic inputs. Haptics, as the new approach to interact with virtual environments (VEs), also benefits the game industry to enlarge their market to users who are not previously reachable [5].

The remainder of this paper is structured as follows. Section 2 reviews the applications of serious games with haptic feedback. Section 3 comprehensively introduces available haptic devices, including tactile devices, force feedback devices, hybrid devices and commercial game devices. A summary of available haptic modeling is also presented in section 3. Section 4 discusses the related research challenges and future directions and section 5 concludes the paper.

\section{Applications}

Applying haptics in serious gaming introduces an additional dimension of perception. For some applications, haptic feedback is purely for improving user experience; some applications provide real-time haptic feedback for users to evaluate their performances during the tasks to enhance training effect, where haptics acts as an auxiliary function; others use it as an alternative of vision or a substitute of motor, haptics plays a main role in these applications. For the first type, discussions about how haptics im- 
prove immersive user experience and related applications can be found in previous literature. This review focuses on the latter two types.

One thing in common among the applications reviewed is that they are built on top of virtual simulation games. As stated in [4], the advantages of virtual simulation in education include:

- Applicability to students of all range of ages and levels

- Low cost and risk of complex tasks that may require expensive equipment and dangerous procedures

- Practicality of theories integrated with math, science and technical skills

- Introduction of new methodology of problem solving

- Variety of career fields requiring skills training with high fidelity of simulation

Serious gaming concepts in virtual simulation help develop more engaging gamelike application with stories, rules, scoring evaluation, rewards or collaboration tasks.

\subsection{Applications with Haptics as Cognitive Aux}

Training and learning tasks are processes of cognition. Our brain integrates every channel of input perception to form our own understanding of a new concept, or a new set of motor manipulation. With haptic feedback, it provides intuitive perception to achieve better understanding, because it delivers an improved cognitive process with fully accessible perception of the abstract scene or phenomena. To generate long term memory of new knowledge or motor dexterity, our brain requires repetitive practice. Serious gaming with incentive of repetitiveness has been applied in areas like science education and surgical training from long time ago.

Science Education. Chan and Black [6] investigated the mental acquisition performance based on three different formats of presentation, text only, text with static visuals, and text with haptic enabled animation game. Experiments were designed for middle school students to learn Newtonian mechanics with the three learning formats respectively. The results showed that for difficult tasks, the students who learnt using haptic animation game format outperformed the others. User learning experience was also reported to be easier and more understandable than other formats.

Haptic-featured teaching helped students obtain the abstract concept more easily. Eigenvalues and eigenvector could be seen and felt directly from the physical dynamic experiments designed in [7]. The force in molecular docking could be mapped from real microscopic world to virtual macroscopic environment [8], and haptics helped students construct mental representation of the concept, as well as binding energy and strength of interactions [9].

Surgical Training. Chui et al. presented a computer-game-like surgical training simulator using force feedback joystick, Delta haptic device, wearable motion capture device CyberGlove and haptic feedback actuator CyberGrasp in [10]. Although multiple haptic devices helped improve the accuracy of haptic feedback and visual ren- 
dering, it also easily introduced system instability and asynchronization. The expensive cost of all the devices was not suitable in real application.

A blood management game for orthopedic surgery has been developed in [11]. Besides graphic simulation, the training aid integrated game features as task-oriented time-attack scenarios. It designed four difficulty levels based on blood-loss severity. Collaboration, bonus and performance evaluation were also featured in this game. The task was to stop bleeding by conducting proper rescue procedures or the game ended with over loss of blood. A 6-DOF haptic device was applied to act as surgical tools in the VE. This application set a good example of the full process of designing a surgical training game with integration of haptic devices. It illustrated the detailed game framework, game design specifications and task flowcharts of the simulation system, with the position and functions of haptic device and haptic rendering clearly shown.

A serious game for training on laparoscopic suturing surgery has been presented in [12]. It designed and developed a first prototype for suturing game using a pair of haptic devices. More advanced virtual reality (VR) simulator (see Fig. 1) using double haptic devices has been reported in [13]. Challenges have been raised in simulation of the soft tissues with visual deformation and haptic force feedback with good accuracy.

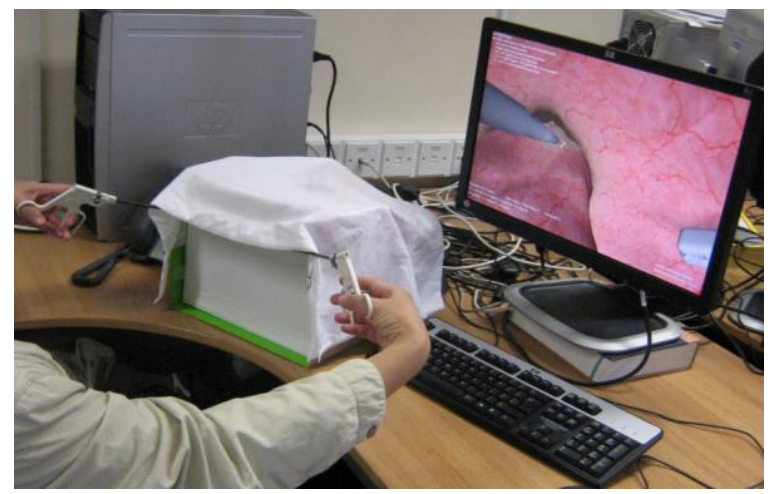

Fig. 1. VR simulator in Laparoscopic Rectum Surgery [13]

\subsection{Applications with Haptics as Main Component}

Human central nervous system takes account of all sensory inputs to generate perception of the environment. One impaired modality can be compensated by other modalities. As for visually impaired users, they can hardly enjoy video games. Haptic enabled games provide them with new experience of gaming. Yuan et al. [14] talked about game accessibility in detail, and offered insights of challenges in future related research.

When the haptic perception is the impaired one, the users are in need of external stimuli to help them recover, so haptics have been widely applied in post stroke rehabilitations. The augmented force helps indicate correctness or incorrectness efficiently in the process of motor training. It also provides guidance force for skills regain. 
Motor Rehabilitation. Fig. 2 shows a game design conceptual model for stroke rehabilitation [15]. Training game examples have been developed based on this taxonomy. They created a VE and connected it to a haptic workbench as the design prototype. By adding new game patterns as shown italicized in Fig. 2, it could easily vary the core game and create new games for different purposes, so to personalize and customize specific rehabilitation game for all variation of training purposes. Using the same concept, a haptic immersive workbench for stroke rehabilitation games has been presented in [16].

Delbressine et al. proposed a novel playful arm-hand rehabilitation using a wearable haptic jacket supporting tilt-sensing and vibrotactile feedback with tabletop interaction [17]. The task of this game was to trap a bug, and the bug would get smaller and more difficult to catch if compensatory movement exceeded a threshold detected by the haptic vest. This design solved the problem of compensatory movements with shoulder and trunk.

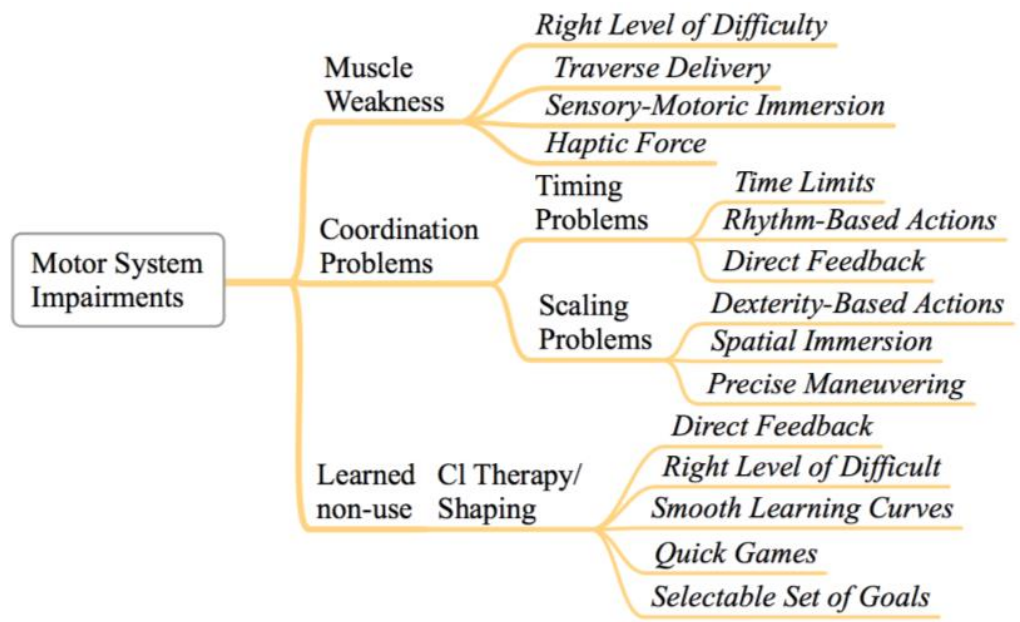

Fig. 2. A subset of the taxonomy [15]

A collaborative game "social maze" has been presented in [18]. They set up a robot-assisted rehabilitation device facilitated with haptic feedback as the central hardware component, allowing patients to train together and interact with therapist.

Haptic handwriting and games have been applied in the rehabilitation system designed in [19]. The haptic Ten Pin bowling game was combined with Novint Falcon haptic device. Handwriting is an essential skill but in a high potential to lose after stroke, since the loss of motor control will harm the complex visuo-motor coordination required by handwriting. Similarly, haptics helped children improve graphmotor skills and grip strength by haptic guidance of the trajectory path [20, 21]. A virtual soccer game was also stated in [22], for children who had walking problems to play soccer with assistance of the haptic driven gait orthosis device.

Henschke et al. presented a new approach for building appropriate serious games for children with cerebral palsy [23]. Children with cerebral palsy lack the tactile 
sensation, so they developed a series of haptic enabled games for this purpose. It focused on a fully accessible game design pattern for providing all available perception for impaired sensory.

Games for Visually Impaired Users. In games for blind or visually impaired users, the main outputs are audio and haptics, some with assistance of salient visual feedback. Existing literature showed satisfied gaming performance.

Yuan and Folmer designed a glove with haptic motor on the tip of each finger for blind users to play Guitar Hero [24]. It interpreted visual stimuli to haptic stimuli by mapping each button to each finger of the glove. The motor would page the user before the corresponding button that needed to be pressed. Nemec et al. [25] inspected the performance of haptic and audio based navigation in VE for visually impaired users. It showed an equal usability with tactile exploration of paper models.

\section{$3 \quad$ Haptic Modeling}

\subsection{Haptic Device}

A typical haptic device contains bidirectional haptic input and output interfaces/sensors between the device and user. Compared with visual feedback, haptic devices require much higher refresh rate $(\geq 1000 \mathrm{~Hz})$ to achieve continuous and real-time perception [26].

Tactile Devices. Tactile Devices simulates temperature, texture, pressure, puncture, friction, roughness, shape that are perceived by cutaneous receptors under our skin. The tactile sensory is generated by actuators which can be developed in different types, mostly are electromagnetic, Shape Memory Alloy (SMA) wire, motor, pneumatic, piezoelectric, polymeric, and Electro Rheological Fluid (ERF). As stated in [27], the most popular technology is electromagnetic and the SMA wire actuation. However, each brings drawbacks in application, weight reduces the portability of electromagnetic actuated devices and low bandwidth limits the performance of SMA. A typical tactile device consists of a set of tactile elements/pins. Various spatial topologies of the pins have been proposed. Pin-matrix is most common as shown in [2833]. Some are shown in the form of a ring [34]. The number of pins, frequency and the pin density are the main parameters concerned in design. Higher density and frequency brings smoother user experience [35]. The current focus of tactile devices research is to simplify system design and materials, to reduce the number of actuators, weight and cost to achieve equally good performance [36-38]. Non-contact tactile interfaces are emerging. An ultrasound-based tactile display using the principles of acoustic radiation pressure and hand tracking was proposed in [39]. It has achieved vibration up to $1 \mathrm{kHz}$ for a volume of $\mathrm{L} 51 \times \mathrm{W} 26 \times \mathrm{H} 19 \mathrm{~mm}^{3}$ space. 
Force Feedback Devices. The force feedback devices can be also called kinesthetic devices, which emulate motion, force, location and compliance that perceived by receptors in our muscles, tendons and joints. A detailed table of commercial force feedback hardware manufactures and devices has been given in [2]. It described the detailed specification of the most prominent devices either in commercial or research.

Similarly with tactile devices, force feedback devices also need one or multiple electromechanical transducers to generate force feedback. Common actuation types include DC motors, electromagnetic, piezoelectric, ERF, and pneumatic etc. Force feedback devices can be divided into grounded and ungrounded categories based on their portability. The popular devices such as SensAble Phantom product, Novint Falcon, Omega, Delta and Virtuose are grounded devices. Ungrounded devices such as the Immersion CyberGrasp glove and the Rutgers Master II [40] come with the shape of gloves. Others are shaped as exoskeleton arm [41, 42].

For most human perception experiment, force feedback need not be set too large (maximum force output of 2-3N can be sufficient), but some rehabilitation exercises expect large force output and workspace.

A dilemma of force feedback devices design exists between high stiffness and low mass since higher stiffness requests larger mass of the material which is normally metal. Portability is limited by the grounded design and large weight. Better performance also associates with larger workspace and more DOF, which leads to higher price. Trade-offs between functional requirements and budget restriction can be analyzed when selecting the proper device.

Hybrid Devices. Hybrid haptic devices incorporate tactile and force feedback but also introduce design challenges from both, as well as synchronization issues and greater complexity of the hardware.

In general, the structure of a hybrid device is implemented by attaching the tactile elements on the end effector of the force feedback device. By manipulating the end effector, the tactile elements will move along with it and provide tactile feedback. An early implementation of hybrid multimodality device is designed based on a mouse [43]. It applied a solenoid pin (tactile element) resting on the mouse button providing tactile feedback for index finger and an electromagnet on the bottom of the mouse paired with an iron mouse pad that providing drag feedback. However, the mouse model is restricted by the low DOF. With force feedback devices that featured with higher than 3-DOF, a thimble with tactile element built-in is attached on the end effector. Users can insert one of their fingers into the thimble to gain both tactile and force feedback [44]. With the restriction of space and the concern of not interfering the force feedback functions, the functionalities of the tactile components on hybrid devices are relatively simpler compared to pure tactile devices. Only one actuator can be fitted into the thimble, so it can only provide single perception. Consequently, thimble design attracted lots of research attention. Kuchenbecker et al. [45] proposed a design that could detect contact location on the fingertip arc, Kuchenbecker et al. [46] had a novel design of the thimble structure for shape and surface recognition. A more complex pin-matrix tactile display component was integrated with a robotic arm WAM (Whole-Arm Manipulator) for simulation of deformable surface [47]. 
Haptic Devices for Entertainment and Gaming. Haptic feedback in gaming is devoted to providing immersive game experience but with more affordable price and portability for common acceptance compared with the research oriented haptic devices. More specifically, to reduce extra cost, vibration actuators have been integrated with the variety of game controllers. Distinguished by their shapes, gaming haptic devices normally appear as mice, joysticks, game pads, vest/jackets, wheels, mobile phones etc.

Microsoft's Explorer Touch Mouse and Arc Touch Mouse both provided light vibration that signals scrolling speed [48]. An optical mouse with friction feedback has been proposed in [49]. As in [43, 50], there was a magnetic iron pad underlays for energizing the mouse with electromagnet to generate 2-DOF force feedbacks.

Game pads/controllers are very common nowadays as XBOX, Wii or PlayStation dominate the game console markets. Feedbacks as rumble or vibration have become standard built-in features in these game controllers. For specific games the controller can be transformed to adapt the game environment by embedding it into extra accessories. For example, the Wii controller comes as a cuboid originally, with Wii Wheel it can be turned into a wheel for racing games, while Wii Zapper turns the controller into a snapshotting gun [51]. Joysticks can either be an individual gadget or integrated with gamepads. A haptic one provides 2-DOF force feedbacks that can mimic gear stick for controlling vehicles and planes [52].

Haptic vest/jackets are emerging gaming devices in recent years. TN Games commercialized the 3RD Space Vest [53] while Saurabh Palan et al. demonstrated a Tactile Gaming Vest (TGV) [54]. Both of them simulated the gunshot feedback by arranging tactile elements around the vest. TGV used solenoids instead of pneumatic to provide a faster experience with shorter responding time.

Haptic steering wheels were specifically for racing games or driving simulation as discussed in $[55,56]$. There were also haptic chairs and seating pads incorporated into gaming.

With the development and popularization of smart phones, mobile games became graphically intense and the prevailing phone platforms also provided haptic feedback interfaces for both hardware and SDK.

Haptic devices mentioned in [2] have also been utilized in game development but not commercially viable due to their high cost. One exception would be Novint Falcon, which was originally designed for video games but also used in academic research because of its high cost-effectiveness.

\subsection{Haptic Modeling}

The definition of haptic modeling has been given in [57], "a series of processes to create haptic content on graphic models that are components of virtual reality, augmented reality, or mixed reality".

Various haptic modeling libraries are available as listed in Table 1. These libraries or toolkits provide programming interfaces (API) for rapid prototyping. Most of them come with not only haptic but also graphic components using either OpenGL or DirectX. Normally commercial haptic hardware will come with SDKs that are only ap- 
plicable to their own devices. For example, OpenHaptics toolkit is only viable for SensAble devices that are the most popular haptic products; Virtual Hand is specifically for the CyberGlove Systems hand tracking devices. Some libraries have specifically been developed hardware independent to adapt more devices. Some provide virtual device adaptation for simulation without requiring a real device connected. Reachin API, HAPTIK and Virtual Hand have network support built in which enables haptic interaction between users. HaptX is designed for game haptics, especially Novint Falcon. The libraries will provide relatively basic haptic features, but they also offer extensibility for customized physical modeling, shape rendering, force effects, collision detection, dynamics and other third-party engines. More haptic modeling libraries are available but not widely applied as the ones listed in Table 1 [57-61].

Table 1. Common Available Haptic Modeling Libraries

\begin{tabular}{|c|c|c|c|c|c|c|}
\hline Name & Devices & $\begin{array}{l}\begin{array}{l}\text { Lan- } \\
\text { guages }\end{array} \\
\end{array}$ & Network & Graphics & $\begin{array}{l}\text { Open- } \\
\text { source }\end{array}$ & Platform \\
\hline $\begin{array}{l}\text { OpenHap- } \\
\text { tics [62] }\end{array}$ & SensAble & $\mathrm{C}++$ & No & Yes & No & $\begin{array}{l}\text { Windows, } \\
\text { Linux }\end{array}$ \\
\hline $\begin{array}{l}\text { CHAI 3D } \\
{[63]}\end{array}$ & $\begin{array}{l}\text { Hardware Inde- } \\
\text { pendent, Virtual } \\
\text { Device }\end{array}$ & $\mathrm{C}++$ & No & Yes & Yes & $\begin{array}{l}\text { Windows, } \\
\text { Linux, Mac }\end{array}$ \\
\hline $\begin{array}{l}\text { H3DAPI } \\
{[64]}\end{array}$ & $\begin{array}{l}\text { SensAble, Novint, } \\
\text { Force Dimension, } \\
\text { MOOG FCS, } \\
\text { G-Coder Systems }\end{array}$ & $\begin{array}{l}\text { X3D, } \\
\text { C++, } \\
\text { Python }\end{array}$ & No & Yes & Yes & $\begin{array}{l}\text { Windows, } \\
\text { Linux, Mac }\end{array}$ \\
\hline $\begin{array}{l}\text { Reachin } \\
\text { API [65] }\end{array}$ & $\begin{array}{l}\text { Hardware Inde- } \\
\text { pendent }\end{array}$ & $\begin{array}{l}\text { C++, } \\
\text { VRML, } \\
\text { Python }\end{array}$ & Yes & Yes & No & Windows \\
\hline HaptX[66] & Novint, SensAble & $\mathrm{C}++$ & No & Yes & No & Windows \\
\hline $\begin{array}{l}\text { HAPTIK } \\
{[67]}\end{array}$ & $\begin{array}{l}\text { Hardware Inde- } \\
\text { pendent (need } \\
\text { related plugin) }\end{array}$ & $\begin{array}{l}\text { C++, } \\
\text { Java, } \\
\text { Matlab, } \\
\text { Sim- } \\
\text { ulink }\end{array}$ & Yes & No & Yes & $\begin{array}{l}\text { Windows, } \\
\text { Linux, Mac }\end{array}$ \\
\hline $\begin{array}{l}\text { Virtual } \\
\text { Hand }\end{array}$ & $\begin{array}{l}\text { CyberGlove Sys- } \\
\text { tems, Virtual } \\
\text { Hand }\end{array}$ & $\mathrm{C}++$ & Yes & Yes & No & Windows \\
\hline
\end{tabular}

By investigation of the available libraries, an abstract architecture of haptic modeling is shown in Fig. 3. At the lower level, the interface for different devices should be provided, or the library is customized to be hardware independent. At the top level, programming utilities are necessary, like wrappers for programming languages that are different with the coding language of the library itself will be provided for adaption of various development environments. Force rendering components are the core of haptic modeling libraries and it is triggered by collision detection component that will also work on graphics components. For lightweight libraries without graphics 
rendering, there must be extendible interfaces provided. For different purposes, the library can be tailored for specific applications. Each component comes with customization abilities for implementing user-defined functions.

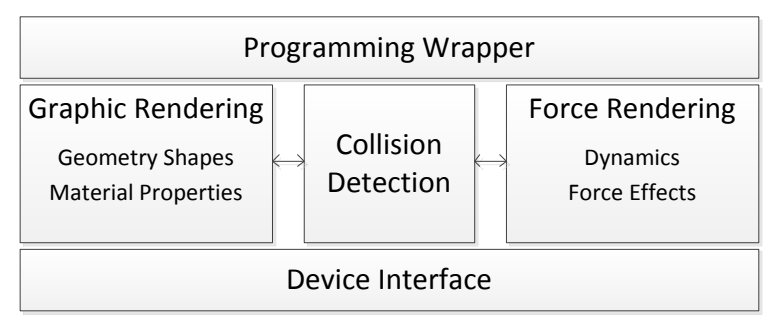

Fig. 3. General Architecture of Haptic Modeling

\section{$4 \quad$ Research Challenges}

From the perspective of what we are focusing in this paper, the challenges for integration of haptics into serious gaming are similar to the challenges exist in other haptic applications. Common issues in haptic rendering has been discussed in [68].

One of the concerns attracts most attention is the computational latency of haptic rendering. The time complexity of haptic rendering algorithms increases with the more complicated scenarios, which designed to simulate Six or higher DOFs and deformable or viscous object/environment [69]. Although the computing techniques and hardware have been improved significantly these days, it is still not good enough for haptic rendering of complicated virtual environment interaction, nor are the existing physical models efficient enough to compensate computational delay [70-73]. Trade-off still exists between computational resources and real-time performance.

In terms of different game specification, different issues emerge. In training games and therapy sessions that have collaboration between experts-novices or physicianspatients, it requires local or network based haptic interactivity support for a shared virtual environment (SVE). Multiuser introduces multiple collision and mutual force impact, which means even more loaded computational task. Haptic data compression and transmission latency are additional issues brought by networks. Some efforts have been made to solve this problem [74-76].

Another major issue is the accuracy of eye-hand coordination, which is the training objective in some rehabilitation applications, for example, writing skills. Occlusion between virtual objects and real objects (hand/tool) is one of the obstacles that prevent getting a better user experience of eye-hand coordination. Techniques as chroma-key and head tracking have been applied for eliminating occlusion [77-79].

Even more sensory modalities have been integrated into VR. It is stated that in a haptic featured environment, sound generated by physical modeling and auditory synthesis techniques can highly improve fidelity [80]. The difficulty would be synchronization of sound and the impact force that caused it. 


\section{Conclusion}

Serious games based on virtual reality share same technology basis with virtual simulation for both hardware and software development. Serious gaming introduces the entertainment and pedagogy features into virtual reality, which marks the main difference. Based on the overview given in this paper, haptics bring advantages to serious gaming in three ways: (1) introduce one more dimension of sensory modality for a more immersive game experience; (2) enhance cognitive process with one more layer of proprioception; (3) augment or compensate impaired perception system.

Challenges are confronted for both game designer and haptic engineer. Game design for education purpose requires psychological knowledge of cognition; it is necessary to figure out what cognitive process benefits from haptic modality and how the game characteristics can be applied for an optimal learning result. Haptic technology still has bottlenecks to be improved. Hardware models are mostly bulky and expensive; rendering algorithms need to be more computational effective to cope with complex VEs. It is believed that haptics in serious games will request interactivity, multimodality and portability.

\section{References}

1. Dondlinger, M.J.: Educational video game design: A review of the literature. Journal of Applied Educational Technology 4, 21-31 (2007)

2. Coles, T.R., Meglan, D., John, N.W.: The role of haptics in medical training simulators: a survey of the state of the art. Haptics, IEEE Transactions on 4, 51-66 (2011)

3. Rego, P., Moreira, P.M., Reis, L.P.: Serious games for rehabilitation: A survey and a classification towards a taxonomy. In: Information Systems and Technologies (CISTI), 2010 5th Iberian Conference on, 1-6. IEEE, (2010)

4. Kincaid, J.P., Westerlund, K.K.: Simulation in education and training. In: Simulation Conference (WSC), Proceedings of the 2009 Winter, 273-280. IEEE, (2009)

5. Derryberry, A.: Serious games: online games for learning. Adobe Whitepaper, November (2007)

6. Chan, M.S., Black, J.B.: Learning Newtonian mechanics with an animation game: The role of presentation format on mental model acquisition. In: Annual Meeting of the American Educational Research Association (AERA), San Francisco. Citeseer, (2006)

7. Okamura, A.M., Richard, C., Cutkosky, M.R.: Feeling is believing: Using a forcefeedback joystick to teach dynamic systems. JOURNAL OF ENGINEERING EDUCATION-WASHINGTON- 91, 345-350 (2002)

8. Krenek, A., Cernohorsky, M., Kabelác, Z., Ac, Z.K.: Haptic visualization of molecular model. (1999)

9. Persson, P.B., Cooper, M.D., Tibell, L.A.E., Ainsworth, S., Ynnerman, A., Jonsson, B.H.: Designing and Evaluating a Haptic System for Biomolecular Education. In: Virtual Reality Conference, 2007. VR '07. IEEE, 171-178. (2007)

10. Chui, C.-K., Ong, J.S., Lian, Z.-Y., Wang, Z., Teo, J., Zhang, J., Yan, C.-H., Ong, S.-H., Wang, S.-C., Wong, H.-K.: Haptics in computer-mediated simulation: Training in vertebroplasty surgery. Simulation \& Gaming 37, 438-451 (2006) 
11. Jing, Q., Yim-Pan, C., Wai-Man, P., Choi, K.-S., Pheng-Ann, H.: Learning Blood Management in Orthopedic Surgery through Gameplay. Computer Graphics and Applications, IEEE 30, 45-57 (2010)

12. De Paolis, L.T.: Serious Game for Laparoscopic Suturing Training. In: Complex, Intelligent and Software Intensive Systems (CISIS), 2012 Sixth International Conference on, 481-485. IEEE, (2012)

13. Pan, J.J., Chang, J., Yang, X., Zhang, J.J., Qureshi, T., Howell, R., Hickish, T.: Graphic and haptic simulation system for virtual laparoscopic rectum surgery. The International Journal of Medical Robotics and Computer Assisted Surgery 7, 304-317 (2011)

14. Yuan, B., Folmer, E., Harris, F., Jr.: Game accessibility: a survey. Universal Access in the Information Society 10, 81-100 (2011)

15. Goude, D., Björk, S., Rydmark, M.: Game design in virtual reality systems for stroke rehabilitation. Studies in health technology and informatics 125, 146-148 (2007)

16. Broeren, J., Sunnerhagen, K.S., Rydmark, M.: Haptic virtual rehabilitation in stroke: transferring research into clinical practice. Physical Therapy Reviews 14, 322-335 (2009)

17. Delbressine, F., Timmermans, A., Beursgens, L., de Jong, M., van Dam, A., Verweij, D., Janssen, M., Markopoulos, P.: Motivating arm-hand use for stroke patients by serious games. In: Conference proceedings:... Annual International Conference of the IEEE Engineering in Medicine and Biology Society. IEEE Engineering in Medicine and Biology Society. Conference, 3564-3567. (2012)

18. De Weyer, T., Robert, K., Renny Octavia Hariandja, J., Alders, G., Coninx, K.: The Social Maze: A Collaborative Game to Motivate MS Patients for Upper Limb Training. Entertainment Computing-ICEC 2012 476-479 (2012)

19. Xu, Z., Yu, H., Yan, S.: Motor rehabilitation training after stroke using haptic handwriting and games. In: Proceedings of the 4th International Convention on Rehabilitation Engineering \& Assistive Technology, 31. Singapore Therapeutic, Assistive \& Rehabilitative Technologies (START) Centre, (2010)

20. Pernalete, N., Edwards, S., Gottipati, R., Tipple, J., Kolipakam, V., Dubey, R.V.: Eyehand coordination assessment/therapy using a robotic haptic device. In: Rehabilitation Robotics, 2005. ICORR 2005. 9th International Conference on, 25-28. (2005)

21. Pernalete, N., Tang, F., Chang, S.M., Cheng, F.Y., Vetter, P., Stegemann, M., Grantner, J.: Development of an evaluation function for eye-hand coordination robotic therapy. In: Rehabilitation Robotics (ICORR), 2011 IEEE International Conference on, 1-6. (2011)

22. Brütsch, K., Schuler, T., Koenig, A., Zimmerli, L., Mérillat, S., Lünenburger, L., Riener, R., Jäncke, L., Meyer-Heim, A.: Influence of virtual reality soccer game on walking performance in robotic assisted gait training for children. (2010)

23. Henschke, M., Hobbs, D., Wilkinson, B.: Developing serious games for children with cerebral palsy: case study and pilot trial. In: Proceedings of the 24th Australian ComputerHuman Interaction Conference, 212-221. ACM, (2012)

24. Yuan, B., Folmer, E.: Blind hero: enabling guitar hero for the visually impaired. Proceedings of the 10th international ACM SIGACCESS conference on Computers and accessibility, 169-176. ACM, Halifax, Nova Scotia, Canada (2008)

25. Nemec, V., Sporka, A., Slavik, P.: Haptic and Spatial Audio Based Navigation of Visually Impaired Users in Virtual Environment Using Low Cost Devices. In: Stary, C., Stephanidis, C. (eds.) User-Centered Interaction Paradigms for Universal Access in the Information Society, vol. 3196, 452-459. Springer Berlin Heidelberg (2004)

26. Hayward, V., Astley, O.R., Cruz-Hernandez, M., Grant, D., Robles-De-La-Torre, G.: Haptic interfaces and devices. Sensor Review 24, 16-29 (2004) 
27. Benali-Khoudja, M., Hafez, M., Alexandre, J.-M., Kheddar, A.: Tactile interfaces: a stateof-the-art survey. In: Int. Symposium on Robotics. (2004)

28. Benali-Khoudja, M., Hafez, M., Kheddar, A.: VITAL: An electromagnetic integrated tactile display. Displays 28, 133-144 (2007)

29. Hasser, C., Weisenberger, J.M.: Preliminary evaluation of a shape memory alloy tactile feedback display. In: Proceedings of the ASME Winter Annual Meeting, Symposium on Haptic Interfaces for Virtual Environments and Teleoperator Systems. (1993)

30. Tan, H.Z., Pentland, A.: Tactual displays for wearable computing. Personal Technologies 1, 225-230 (1997)

31. Pawluk, D.T., Van Buskirk, C., Killebrew, J., Hsiao, S., Johnson, K.: Control and pattern specification for a high density tactile array. In: IMECE Proc. of the ASME Dyn. Sys. and Control Div, 97-102. (1998)

32. Ikei, Y., Yamada, M., Fukuda, S.: A new design of haptic texture display-Texture Display2-and its preliminary evaluation. In: Virtual Reality, 2001. Proceedings. IEEE, 2128. IEEE, (2001)

33. Wagner, C.R., Lederman, S.J., Howe, R.D.: A tactile shape display using RC servomotors. In: Haptic Interfaces for Virtual Environment and Teleoperator Systems, 2002. HAPTICS 2002. Proceedings. 10th Symposium on, 354-355. IEEE, (2002)

34. Sato, K., Igarashi, E., Kimura, M.: Development of non-constrained master arm with tactile feedback device. In: Advanced Robotics, 1991.'Robots in Unstructured Environments', 91 ICAR., Fifth International Conference on, 334-338. IEEE, (1991)

35. Watanabe, T., Fukui, S.: A method for controlling tactile sensation of surface roughness using ultrasonic vibration. In: Robotics and Automation, 1995. Proceedings., 1995 IEEE International Conference on, 1134-1139. IEEE, (1995)

36. Benali-Khoudja, M., Beny, A.L., Hafez, M., Kheddar, A.: VT vector-touch: a new slip/stretch tactile display. In: Intelligent Robots and Systems, 2004. (IROS 2004). Proceedings. 2004 IEEE/RSJ International Conference on, 3583-3588 vol.3584. (2004)

37. Chinello, F., Malvezzi, M., Pacchierotti, C., Prattichizzo, D.: A three DoFs wearable tactile display for exploration and manipulation of virtual objects. In: Haptics Symposium (HAPTICS), 2012 IEEE, 71-76. (2012)

38. Marquardt, N., Nacenta, M.A., Young, J.E., Carpendale, S., Greenberg, S., Sharlin, E.: The Haptic Tabletop Puck: tactile feedback for interactive tabletops. Proceedings of the ACM International Conference on Interactive Tabletops and Surfaces, 85-92. ACM, Banff, Alberta, Canada (2009)

39. Hoshi, T.: Development of aerial-input and aerial-tactile-feedback system. In: World Haptics Conference (WHC), 2011 IEEE, 569-573. (2011)

40. Bouzit, M., Burdea, G., Popescu, G., Boian, R.: The Rutgers Master II-new design forcefeedback glove. Mechatronics, IEEE/ASME Transactions on 7, 256-263 (2002)

41. Chen, E., Eberman, B., Marcus, B.A.: Method and Apparatus to Create a Complex Tctile Sensation. Microsoft Corporation, US (1998)

42. Frisoli, A., Rocchi, F., Marcheschi, S., Dettori, A., Salsedo, F., Bergamasco, M.: A new force-feedback arm exoskeleton for haptic interaction in virtual environments. In: Eurohaptics Conference, 2005 and Symposium on Haptic Interfaces for Virtual Environment and Teleoperator Systems, 2005. World Haptics 2005. First Joint, 195-201. (2005)

43. Mackenzie, I.S.: Movement characteristics using a mouse with tactile and force feedback. Int. J. Human-Computer Studies 45, 483-493 (1996) 
44. Hasser, C.J., Daniels, M.W.: Tactile feedback with adaptive controller for a forcereflecting haptic display. 1. Design. In: Biomedical Engineering Conference, 1996., Proceedings of the 1996 Fifteenth Southern, 526-529. (1996)

45. Kuchenbecker, K.J., Provancher, W.R., Niemeyer, G., Cutkosky, M.R.: Haptic display of contact location. In: Haptic Interfaces for Virtual Environment and Teleoperator Systems, 2004. HAPTICS '04. Proceedings. 12th International Symposium on, 40-47. (2004)

46. Kuchenbecker, K.J., Ferguson, D., Kutzer, M., Moses, M., Okamura, A.M.: The Touch Thimble: Providing Fingertip Contact Feedback During Point-Force Haptic Interaction. In: Haptic interfaces for virtual environment and teleoperator systems, 2008. haptics 2008. symposium on, 239-246. (2008)

47. Wagner, C.R., Perrin, D.P., Feller, R.L., Howe, R.D., Clatz, O., Delingette, H., Ayache, N.: Integrating Tactile and Force Feedback with Finite Element Models. In: Robotics and Automation, 2005. ICRA 2005. Proceedings of the 2005 IEEE International Conference on, 3942-3947. (2005)

48. Microsoft, http://www.microsoft.com/hardware/en-gb/touch-technology

49. Schneider, C., Mustufa, T., Okamura, A.: A magnetically-actuated friction feedback mouse. Proceedings of EuroHaptics 2004, Munich, Allemagne 330-337 (2004)

50. Wanjoo, P., Sehyung, P., Laehyun, K., Seungjae, S.: Haptic Mouse Interface Actuated by an Electromagnet. In: Complex, Intelligent and Software Intensive Systems (CISIS), 2011 International Conference on, 643-646. (2011)

51. Nintendo, http://www.nintendo.com/wii/enhance/\#/accessories

52. Orozco, M., Silva, J., El Saddik, A., Petriu, E.: The Role of Haptics in Games. Haptics Rendering and Applications, Abdulmotaleb El Saddik (Ed.), ISBN 978-953 (2012)

53. TN Games, http://tngames.com/

54. Palan, S.: Tactile Gaming Vest (TGV). http://iroboticist.com/2010/03/26/tgv/ (2010)

55. Mohellebi, H., Kheddar, A., Espie, S.: Adaptive Haptic Feedback Steering Wheel for Driving Simulators. Vehicular Technology, IEEE Transactions on 58, 1654-1666 (2009)

56. Sungjae, H., Jung-hee, R.: The Haptic steering Wheel: Vibro-tactile based navigation for the driving environment. In: Pervasive Computing and Communications Workshops (PERCOM Workshops), 2010 8th IEEE International Conference on, 660-665. (2010)

57. Ryu, J., Kim, H.: A Haptic Modeling System (2010)

58. Ott, R., De Perrot, V., Thalmann, D., Vexo, F.: MHaptic : a Haptic Manipulation Library for Generic Virtual Environments. In: Cyberworlds, 2007. CW '07. International Conference on, 338-345. (2007)

59. ANDERSON, T.G., Breckenridge, A., DAVIDSON, G.S.: FGB: a graphical and haptic user interface for creating graphical, haptic user interfaces. Sandia National Labs., Albuquerque, NM (US); Sandia National Labs., Livermore, CA (US) (1999)

60. Yongwon, S., Beom-Chan, L., Yeongmi, K., Jong-Phil, K., Ryu, J.: K-HapticModeler ${ }^{\mathrm{TM}}$ : a haptic modeling scope and basic framework. In: Haptic, Audio and Visual Environments and Games, 2007. HAVE 2007. IEEE International Workshop on, 136-141. (2007)

61. Eid, M., Andrews, S., Alamri, A., Saddik, A.: HAMLAT: A HAML-Based Authoring Tool for Haptic Application Development. In: Ferre, M. (ed.) Haptics: Perception, Devices and Scenarios, vol. 5024, 857-866. Springer Berlin Heidelberg (2008)

62. SensAbleTechnologies, http://www.sensable.com/documents/documents/OpenHaptics_datasheet_hi.pdf

63. Conti, F., Barbagli, F., Balaniuk, R., Halg, M., Lu, C., Morris, D., Sentis, L., Vileshin, E., Warren, J., Khatib, O.: The CHAI libraries. In: Proceedings of Eurohaptics 2003, 496-500. (2003)

64. SenseGraphics, http://www.sensegraphics.com/datasheet/H3DAPI_datasheet.pdf 
65. ReachinTechnologies, http://www.reachin.se/products/ReachinAPI/

66. ReachinTechnologies, http://www.haptx.com

67. De Pascale, M., Prattichizzo, D.: The Haptik Library: A Component Based Architecture for Uniform Access to Haptic Devices. Robotics \& Automation Magazine, IEEE 14, 64-75 (2007)

68. McLaughlin, M.L., Hespanha, J.P., Sukhatme, G.S.: Introduction to haptics. Prentice-Hall (2002)

69. Otaduy, M.A., Lin, M.C.: Introduction to haptic rendering. ACM SIGGRAPH 2005 Courses, 3. ACM, Los Angeles, California (2005)

70. Bathe, K.-J.: Finite element procedures. Prentice hall Englewood Cliffs, NJ (1996)

71. Teran, J., Blemker, S., Hing, V., Fedkiw, R.: Finite volume methods for the simulation of skeletal muscle. In: Proceedings of the 2003 ACM SIGGRAPH/Eurographics symposium on Computer animation, 68-74. Eurographics Association, (2003)

72. James, D.L., Pai, D.K.: Multiresolution green's function methods for interactive simulation of large-scale elastostatic objects. ACM Transactions on Graphics (TOG) 22, 47-82 (2003)

73. Chang, J., Yang, X., Pan, J.J., Li, W., Zhang, J.J.: A fast hybrid computation model for rectum deformation. The Visual Computer 27, 97-107 (2011)

74. Basdogan, C., Ho, C.-H., Srinivasan, M.A., Slater, M.: An experimental study on the role of touch in shared virtual environments. ACM Trans. Comput.-Hum. Interact. 7, 443-460 (2000)

75. Kim, J., Kim, H., Tay, B.K., Muniyandi, M., Srinivasan, M.A., Jordan, J., Mortensen, J., Oliveira, M., Slater, M.: Transatlantic Touch: A Study of Haptic Collaboration over Long Distance. Presence: Teleoperators and Virtual Environments 13, 328-337 (2004)

76. Liu, G., Lu, K.: Networked multiplayer cooperative interaction using decoupled motion control method in a shared virtual environment with haptic, visual and movement feedback. Computer Animation and Virtual Worlds (2012)

77. Yokokohji, Y., Hollis, R.L., Kanade, T.: What you can see is what you can feeldevelopment of a visual/haptic interface to virtual environment. In: Virtual Reality Annual International Symposium, 1996., Proceedings of the IEEE 1996, 46-53, 265. IEEE, (1996)

78. Inami, M., Kawakami, N., Sekiguchi, D., Yanagida, Y., Maeda, T., Tachi, S.: Visuo-haptic display using head-mounted projector. In: Virtual Reality, 2000. Proceedings. IEEE, 233240. (2000)

79. Arsenault, R., Ware, C.: Eye-hand co-ordination with force feedback. In: Proceedings of the SIGCHI conference on Human factors in computing systems, 408-414. ACM, (2000)

80. Avanzini, F., Crosato, P.: Haptic-Auditory Rendering and Perception of Contact Stiffness. In: McGookin, D., Brewster, S. (eds.) Haptic and Audio Interaction Design, vol. 4129, 24 35. Springer Berlin Heidelberg (2006) 